\title{
Differences in the faecal microbiota of children with $\beta$-cell autoimmunity
}

The faecal microbiota of children with $\beta$-cell autoimmunity contains different levels of certain bacteria than that of children without $\beta$-cell autoimmunity, new results show.

Animal studies have previously shown that changes in the intestinal microbiota are associated with the development of autoimmune diabetes mellitus. These findings led a team of researchers to question whether the gut microbiota of children with multiple autoantibodies indicating increased risk of type 1 diabetes mellitus differed from that of children who are predicted to remain healthy.

The investigators took faecal samples from 18 children with two or more diabetes-associated autoantibodies ( $\beta$-cell autoimmunity) and 18 children without autoantibodies matched for age, sex and HLA-DQB1 genotype. "We used 16S rRNA pyrosequencing, which gives a good picture of the composition of the bacterial microbiota," explains author Outi Vaarala.
Children with $\beta$-cell autoimmunity had higher levels of bacteria from the Bacteroidetes phylum, the Bacteroidaceae family and the Bacteroides genus, but lower levels of species that produce lactate and butyrate than children without $\beta$-cell autoimmunity. In addition, the levels of two Bifidobacterium species that are usually dominant in faeces - Bifidobacterium adolescentis and Bifidobacterium pseudocatenulatum-were reduced in stool samples from children with $\beta$-cell autoimmunity.

The authors suggest that a lack of butyrate could be associated with the intestinal immune activation seen in patients with type 1 diabetes mellitus, but note that more work is needed to understand the role of the Bacteroides.

Claire Greenhill

Original article de Goffau, M. C. et al. Fecal microbiota composition differs between children with $\beta$-cell autoimmunity and those without. Diabetes doi:10.2337/db12-0526 